\title{
Alpha-fair resource allocation under incomplete information and presence of a jammer *
}

\author{
Eitan Altman ${ }^{1}$, Konstantin Avrachenkov ${ }^{1}$, and Andrey Garnaev ${ }^{2}$ \\ 1 INRIA Sophia Antipolis, France \\ \{altman, k. avrachenkov\}@sophia.inria.fr \\ 2 St. Petersburg State University, Russia \\ agarnaev@rambler.ru
}

\begin{abstract}
In the present work we deal with the concept of alpha-fair resource allocation in the situation where the decision maker (in our case, the base station) does not have complete information about environment. Namely, we develop a concept of $\alpha$-fairness under uncertainty to allocate power resource in the presence of a jammer under two types of uncertainty: (a) the decision maker does not have complete knowledge about the parameters of the environment, but knows only their distribution, (b) the jammer can come into the environment with some probability bringing extra background noise. The goal of the decision maker is to maximize the $\alpha$-fairness utility function with respect to the SNIR (signal to noise-plus-interference ratio). Here we consider a concept of the expected $\alpha$-fairness utility function (short-term fairness) as well as fairness of expectation (long-term fairness). In the scenario with the unknown parameters of the environment the most adequate approach is a zero-sum game since it can also be viewed as a minimax problem for the decision maker playing against the nature where the decision maker has to apply the best allocation under the worst circumstances. In the scenario with the uncertainty about jamming being in the system the Nash equilibrium concept is employed since the agents have non-zero sum payoffs: the decision maker would like to maximize either the expected fairness or the fairness of expectation while the jammer would like to minimize the fairness if he comes in on the scene. For all the plots the equilibrium strategies in closed form are found. We have shown that for all the scenarios the equilibrium has to be constructed into two steps. In the first step the equilibrium jamming strategy has to be constructed based on a solution of the corresponding modification of the water-filling equation. In the second step the decision maker equilibrium strategy has to be constructed equalizing the induced by jammer background noise.
\end{abstract}

Keywords: Wireless networks, Power Control, Incomplete Information, Nash Equilibrium, Saddle Point

\footnotetext{
* The work was supported by EGIDE ECO-NET grant no.18933SL "Game Theory for Wireless Networks" and RFBR Grant no.09-01-00334-a.
} 


\section{Introduction}

Fairness concepts have been playing a central role in networking. In the ATM standards [9], the maxmin fairness and its weighted versions appear as the way to allocate throughput to connections using the ABR (Available Bit Rate) best effort service. The proportional fairness has been introduced in [5,6]. Later it was implemented in wireless communications (e.g. in the Qualcomm High Data Rate (HDR) scheduler) as a way to allocate throughputs (through time slots); it has also been shown to correspond to the way that some versions of the TCP Internet Protocol share bottleneck capacities [8]. A unifying mathematical formulation to fair throughput assignment (which we call the " $\alpha$-fairness") has been proposed in [7].

In the present work we deal with the concept of alpha-fair resource allocation in the situation where the decision maker (in our case, a base station) does not have complete information about environment. Namely, we develop a concept of $\alpha$-fairness under uncertainty to allocate power resource in the presence of a jammer under two types of uncertainty: (a) the decision maker does not have complete knowledge about the parameters of the environment but knows only their distribution, (b) the jammer can come into the environment with some probability bringing extra background noise. These scenarios have not been considered previously in the literature (see e.g., [1-4] and references therein). The goal of the decision maker is to maximize the $\alpha$-fairness utility function with respect to the SNIR (signal to noise-plus-interference ratio). Here we consider a concept of the expected fairness as well as fairness of expectation. The expected fairness concept is appropriate for the case of slow fading when the decision maker dynamics is faster than the evolution of the environment. Whereas the fairness of expectation concept is more appropriate for the case of fast fading when the state of the environment changes quicker than the dynamics of the control system. We can also say that the expected fairness can be used as a short-term fairness concept while the fairness of expectation can be applicable for long-term fairness.

In the scenario with the unknown parameters of the environment the most adequate approach is a zero-sum game since it can also be viewed as a minimax problem for the decision maker playing against the nature where the decision maker has to apply the best allocation under the worst circumstances. In the scenario with the uncertainty about jamming being in the system the Nash equilibrium concept is employed since the agents have non-opposite goals: the decision maker would like to maximize either the expected fairness or the fairness of expectation while the jammer would like to minimize the fairness if he comes in on the scene. For all the plots the equilibrium solutions are found in closed form.

\section{Dependent fading channel gains: expected fairness}

In this section we consider the following power resource allocation problem. There is a single decision maker (say, the base station) that decides how to al- 
locate the power between $n$ different users. The base station transmits to the mobiles simultaneously using independent channels, e.g. different directional antennas or frequency bands (e.g. as in OFDM, where one should assign different power levels for different sub-carriers [10]).

The strategy of the decision maker is $P=\left(P_{1}, \ldots, P_{n}\right)$ such that $P_{i} \geq 0$ for $i \in$ $[1, n]$ and $\sum_{i=1}^{n} P_{i}=\bar{P}$, where $\bar{P}$ is the total power which the decision maker has to distribute among all the users and $P_{i}$ corresponds to a power level assigned to the $i$-th user.

In the environment a jammer could be present bringing extra background noise of the total power $\bar{J}$ to the natural one distributing it among users. So, the pure strategy of the jammer is $J=\left(J_{1}, \ldots, J_{n}\right)$ where $J_{i} \geq 0$ for $i \in$ $[1, n]$ and $\sum_{i=1}^{n} J_{i}=\bar{J}$.

The decision maker does not know the fading channels gains of users with certainty. Namely, the fading channel gains can be random, i.e. with probability $p^{k}, k \in[1, K]$ they are $g_{i}^{k}$ (for the user) and $h_{i}^{k}$ (for the jammer), respectively, $i \in[1, n]$. The uncontrolled noise for user $i$ is $N_{i}^{0 k}$ also with probability $p^{k}$, $k \in[1, K]$. Thus, in this section we assume that the fading channel gains are dependent. The users know with certainty that a jammer is present in the system. As a payoff we consider $\alpha$-fairness $(\alpha>0)$ utility function of the expected SNIRs:

$$
v(P, J)=\frac{1}{1-\alpha} \sum_{k=1}^{K} p^{k} \sum_{i=1}^{n}\left(\frac{g_{i}^{k} P_{i}}{N_{i}^{0 k}+h_{i}^{k} J_{i}}\right)^{1-\alpha} \text { for } \alpha \neq 1
$$

and

$$
v(P, J)=\sum_{k=1}^{K} p^{k} \sum_{i=1}^{n} \ln \left(\frac{g_{i}^{k} P_{i}}{N_{i}^{0 k}+h_{i}^{k} J_{i}}\right) \text { for } \alpha=1,
$$

We assume that all the fading channel gains $g_{i}^{k}, h_{i}^{k}$ and the power level of the uncontrolled noise $N_{i}^{0 k}$ for $i \in[1, n]$, the probabilities $p^{k}$ which the system is in as well as the total power resource $\bar{P}$ of the decision maker and the total noise $\bar{J}$ induced by the jammer are fixed and known to both agents.

In this setting the jammer more naturally has to be interpreted as a natural background noise which is present in the system. The total power of the jammer is known to the decision maker but the decision maker does not know its distribution among the users. The decision maker wants to distribute resources among the users in the worst situation, so it faces a maxmin problem. Thus, here we deal with a zero-sum game, and the payoff to the jammer is $-v(P, J)$. We will look for the saddle point and the value of the game, that is, we want to find the strategies $\left(P^{*}, J^{*}\right) \in A \times B$ such that

$$
v\left(P, J^{*}\right) \leq v\left(P^{*}, J^{*}\right) \leq v\left(P^{*}, J\right) \text { for any }(P, J) \in A \times B,
$$

where $A$ and $B$ are the sets of all the strategies of the decision maker and jammer, respectively and $v=v\left(P^{*}, J^{*}\right)$ is the value of the game and $\left(P^{*}, J^{*}\right)$ is the saddle point. 
Before starting studying our problem introduce the following notation. Denote by $\mathcal{P}$ the decision maker strategy assigning to allocate resources uniformly among all the users, namely, $\mathcal{P}=(\bar{P} / n, \ldots, \bar{P} / n)$.

The next Lemma based on the Kuhn-Tucker Theorem describes the structure of the saddle point.

Lemma 1 Let $\alpha \in(0,1]$ then $(P, J)$ is a saddle point if and only if there are $\omega$ and $\nu$ (Lagrange multipliers) such that

(a) for $0<\alpha<1$

$$
\begin{gathered}
\frac{1}{P_{i}^{\alpha}} \sum_{k=1}^{K} p^{k}\left(\frac{g_{i}^{k}}{N_{i}^{0 k}+h_{i}^{k} J_{i}}\right)^{1-\alpha} \begin{cases}=\omega, & P_{i}>0, \\
\leq \omega, & P_{i}=0,\end{cases} \\
P_{i}^{1-\alpha} \sum_{k=1}^{K} p^{k} \frac{h_{i}^{k}}{N_{i}^{0 k}+h_{i}^{k} J_{i}}\left(\frac{g_{i}^{k}}{N_{i}^{0 k}+h_{i}^{k} J_{i}}\right)^{1-\alpha} \begin{cases}=\nu, & J_{i}>0, \\
\leq \nu, & J_{i}=0,\end{cases}
\end{gathered}
$$

(b) for $\alpha=1$

$$
\begin{gathered}
\frac{1}{P_{i}} \begin{cases}=\omega, & P_{i}>0, \\
\leq \omega, & P_{i}=0,\end{cases} \\
\sum_{k=1}^{K} p^{k} \frac{h_{i}^{k}}{N_{i}^{0 k}+h_{i}^{k} J_{i}} \begin{cases}=\nu, & J_{i}>0, \\
\leq \nu, & J_{i}=0 .\end{cases}
\end{gathered}
$$

First we will study the case with $0<\alpha<1$. From (3) it follows that necessarily $P_{i}>0$ for all $i$. Then

$$
P_{i}=\frac{1}{\omega^{1 / \alpha}}\left(\sum_{k=1}^{K} p^{k}\left(\frac{g_{i}^{k}}{N_{i}^{0 k}+h_{i}^{k} J_{i}}\right)^{1-\alpha}\right)^{1 / \alpha} \text { for } i \in[1, n]
$$

Thus, by (4),

$$
\begin{aligned}
& \left(\sum_{k=1}^{K} p^{k}\left(\frac{g_{i}^{k}}{N_{i}^{0 k}+h_{i}^{k} J_{i}}\right)^{1-\alpha}\right)^{(1-\alpha) / \alpha} \sum_{k=1}^{K} \frac{p^{k} h_{i}^{k}}{N_{i}^{0 k}+h_{i}^{k} J_{i}}\left(\frac{g_{i}^{k}}{N_{i}^{0 k}+h_{i}^{k} J_{i}}\right)^{1-\alpha} \\
& \left\{\begin{array}{l}
=\nu \omega^{(1-\alpha) / \alpha}, \quad J_{i}>0, \\
\leq \nu \omega^{(1-\alpha) / \alpha}, \quad J_{i}=0 .
\end{array}\right.
\end{aligned}
$$

Let

$$
R_{i}(x):=\left(\sum_{k=1}^{K} p^{k}\left(\frac{g_{i}^{k}}{N_{i}^{0 k}+h_{i}^{k} x}\right)^{1-\alpha}\right)^{(1-\alpha) / \alpha} \sum_{k=1}^{K} p^{k} \frac{h_{i}^{k}}{N_{i}^{0 k}+h_{i}^{k} x}\left(\frac{g_{i}^{k}}{N_{i}^{0 k}+h_{i}^{k} x}\right)^{1-\alpha}
$$


It is clear that $R_{i}(x)$ is a continuous and decreasing function on $x \in[0, \infty)$ such that $R_{i}(x) \rightarrow 0$ for $x \rightarrow \infty$. Then we can define $J_{i}(\tau)$ as follows

$$
J_{i}(\tau)= \begin{cases}\text { the unique root of } R_{i}(x)=\tau, & \text { if } R_{i}(0)>\tau \\ 0, & \text { if } R_{i}(0) \leq \tau\end{cases}
$$

It is clear that $J_{i}(x)$ is continuous for $x \in[0, \infty)$ and decreasing while it is positive. Also, $J_{i}(\tau) \rightarrow \infty$ for $\tau \rightarrow 0$, and $J_{i}(x)=0$ for $\tau \geq J_{i}(0)$. Thus, there is the unique $x=\tau^{*}$ such that

$$
\sum_{i=1}^{n} J_{i}(x)=\bar{J}
$$

Thus, we have proved the following result supplying the saddle point strategies:

Theorem 1 Let $0<\alpha<1$ then the saddle point of jammer is given by (8) where $\tau=\tau^{*}$ is the unique root of (9). The saddle point strategy of the decision maker is given as follows

$$
P_{i}=\frac{\left(\sum_{k=1}^{K} p^{k}\left(\frac{g_{i}^{k}}{N_{i}^{0 k}+h_{i}^{k} J_{i}\left(\tau_{*}\right)}\right)^{1-\alpha}\right)^{1 / \alpha}}{\sum_{r=1}^{n}\left(\sum_{k=1}^{K} p^{k}\left(\frac{g_{r}^{k}}{N_{r}^{0 k}+h_{r}^{k} J_{r}\left(\tau_{*}\right)}\right)^{1-\alpha}\right)^{1 / \alpha}} \bar{P} \quad \text { for } i \in[1, n] .
$$

It is interesting about these strategies that in the optimal behavior the jammer can harm only the best sub-carriers while the decision maker uses all of them. Similarly we can study the case where $\alpha=1$, namely, we show in the following theorem that the saddle point jammer strategy has a water-filling structure while the saddle point decision maker strategy has to be the uniform one.

Theorem 2 Let $\alpha=1$ then the saddle point jammer strategy is given as follows

$$
J_{i}(\nu)= \begin{cases}\text { the unique root of } F_{i}(x)=\nu, & \text { if } F_{i}(0)>\nu, \\ 0, & \text { if } F_{i}(0) \leq \nu,\end{cases}
$$

where

$$
F_{i}(x):=\sum_{k=1}^{K} p^{k} \frac{g_{i}^{k}}{N_{i}^{0 k}+h_{i}^{k} x}
$$

and $\nu=\nu^{*}$ is the unique root of $\sum_{i=1}^{n} J_{i}(\nu)=\bar{J}$. The saddle point decision maker strategy is the uniform one $\mathcal{P}$. 


\section{Independent fading channel gains: expected fairness}

In this section we assume that the fading channel gains are independent. Namely, with probability $p^{k}, k \in[1, K]$ the fading channel gain of the jammer on the channel of user $i$ is $h_{i}^{k}$ and the uncontrolled noise for user $i$ is $N_{i}^{0 k}$. With probability $q^{l}, l \in[1, L]$ the fading channel gain of user $i$ is $g_{i}^{l}$. As a payoff we consider the expected $\alpha$-fairness $(\alpha \geq 0)$ utility function of the SNIRs

$$
v(P, J)=\frac{1}{1-\alpha} \sum_{k=1}^{K} \sum_{l=1}^{L} p^{k} q^{l} \sum_{i=1}^{n}\left(\frac{g_{i}^{l} P_{i}}{N_{i}^{0 k}+h_{i}^{k} J_{i}}\right)^{1-\alpha} \text { for } \alpha \neq 1
$$

and

$$
v(P, J)=\sum_{k=1}^{K} \sum_{l=1}^{L} p^{k} q^{l} \sum_{i=1}^{n} \ln \left(\frac{g_{i}^{l} P_{i}}{N_{i}^{0 k}+h_{i}^{k} J_{i}}\right) \text { for } \alpha=1 .
$$

It is clear that (10) is equivalent to

$$
v(P, J)=\frac{1}{1-\alpha} \sum_{k=1}^{K} p^{k} \sum_{i=1}^{n} \bar{g}_{i}\left(\frac{P_{i}}{N_{i}^{0 k}+h_{i}^{k} J_{i}}\right)^{1-\alpha} \text { for } \alpha \neq 1,
$$

where

$$
\bar{g}_{i}=\sum_{l=1}^{L} q^{l}\left(g_{i}^{l}\right)^{1-\alpha} .
$$

Then from Theorem 3 the following result supplying the optimal strategies follows:

Theorem 3 (a) Let $0<\alpha<1$ then the saddle point jammer strategy is given as follows

$$
J_{i}=J_{i}\left(\tau^{*}\right) \text { for } i \in[1, n],
$$

where $\tau^{*}$ is the unique root of $\sum_{i=1}^{n} J_{i}(\tau)=\bar{J}$ and

$$
J_{i}(\tau)= \begin{cases}\text { the unique root of } R_{i}(x)=\tau, & \text { if } R_{i}(0)>\tau \\ 0, & \text { if } R_{i}(0) \leq \tau\end{cases}
$$

where

$$
R_{i}(x):=\bar{g}_{i}^{1 / \alpha-1}\left(\sum_{k=1}^{K} \frac{p^{k}}{\left(N_{i}^{0 k}+h_{i}^{k} x\right)^{1-\alpha}}\right)^{(1-\alpha) / \alpha} \sum_{k=1}^{K} p^{k} \frac{h_{i}^{k}}{\left(N_{i}^{0 k}+h_{i}^{k} x\right)^{2-\alpha}} .
$$

The saddle point decision maker strategy is given as follows

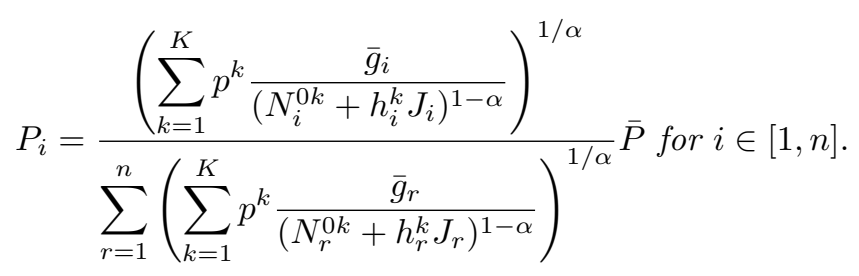


(b) Let $\alpha=1$ then the saddle point jammer strategy is given as follows

$$
J_{i}(\nu)= \begin{cases}\text { the unique root of } F_{i}(x)=\nu, & \text { if } F_{i}(0)>\nu, \\ 0, & \text { if } F_{i}(0) \leq \nu,\end{cases}
$$

where

$$
F_{i}(x):=\sum_{k=1}^{K} p^{k} \frac{\bar{g}_{i}}{N_{i}^{0 k}+h_{i}^{k} x}
$$

and $\nu=\nu^{*}$ is the unique root of $\sum_{i=1}^{n} J_{i}(\nu)=\bar{J}$. The saddle point decision maker strategy is the uniform one in $[1, n]$, namely, it is given as follows $P_{i}=$ $\bar{P} / n$ for $i \in[1, n]$.

\section{Dependent fading channel gains: fairness of expectation}

In this section as a payoff to the decision maker we consider $\alpha$-fairness $(\alpha>0)$ utility function of the expected SNIRs:

$$
v(P, J)=\frac{1}{1-\alpha} \sum_{i=1}^{n}\left(\sum_{k=1}^{K} p^{k} \frac{g_{i}^{k} P_{i}}{N_{i}^{0 k}+h_{i}^{k} J_{i}}\right)^{1-\alpha} \text { for } \alpha \neq 1
$$

and

$$
v(P, J)=\sum_{i=1}^{n} \ln \left(\sum_{k=1}^{K} p^{k} \frac{g_{i}^{k} P_{i}}{N_{i}^{0 k}+h_{i}^{k} J_{i}}\right) \text { for } \alpha=1,
$$

where $\alpha \geq 0$. The next Lemma based on the Kuhn-Tucker Theorem describes the structure of the saddle point.

Lemma 2 Let $\alpha \in(0,1]$ then $(P, J)$ is a saddle point if and only if there are $\omega$ and $\nu$ (Lagrange multipliers) such that

(a) for $0<\alpha<1$

$$
\begin{gathered}
\frac{1}{P_{i}^{\alpha}}\left(\sum_{k=1}^{K} \frac{p^{k} g_{i}^{k}}{N_{i}^{0 k}+h_{i}^{k} J_{i}}\right)^{1-\alpha} \begin{cases}=\omega, & P_{i}>0, \\
\leq \omega, & P_{i}=0,\end{cases} \\
P_{i}^{1-\alpha} \frac{\sum_{k=1}^{K} \frac{p^{k} h_{i}^{k} g_{i}^{k}}{\left(N_{i}^{0 k}+h_{i}^{k} J_{i}\right)^{2}}}{\left(\sum_{k=1}^{K} \frac{p^{k} g_{i}^{k}}{N_{i}^{0 k}+h_{i}^{k} J_{i}}\right)^{\alpha}} \begin{cases}=\nu, & J_{i}>0, \\
\leq \nu, & J_{i}=0,\end{cases}
\end{gathered}
$$

(b) for $\alpha=1$

$$
\begin{gathered}
\frac{1}{P_{i}} \begin{cases}=\omega, & P_{i}>0, \\
\leq \omega, & P_{i}=0,\end{cases} \\
\sum_{k=1}^{K} p^{k} \frac{h_{i}^{k} g_{i}^{k}}{N_{i}^{0 k}+h_{i}^{k} J_{i}} \begin{cases}=\nu, & J_{i}>0, \\
\leq \nu, & J_{i}=0 .\end{cases}
\end{gathered}
$$


The lemma implies the following result supplying the saddle point.

Theorem 4 Let $0<\alpha \leq 1$ then the saddle point decision maker strategy is given as follows

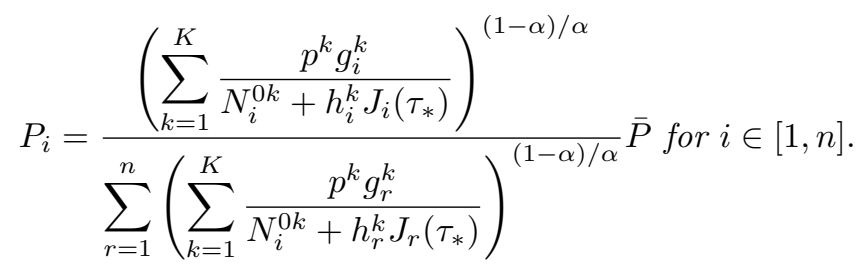

The saddle point jammer strategy is given by

$$
J_{i}(\tau)= \begin{cases}\text { the root of } R_{i}(x)=\tau, & \text { if } R_{i}(0)>\tau, \\ 0, & \text { if } R_{i}(0) \leq \tau .\end{cases}
$$

where

$$
R_{i}(x):=\left(\sum_{k=1}^{K} \frac{p^{k} g_{i}^{k}}{N_{i}^{0 k}+h_{i}^{k} x}\right)^{1 / \alpha-2} \sum_{k=1}^{K} \frac{p^{k} h_{i}^{k} g_{i}^{k}}{\left(N_{i}^{0 k}+h_{i}^{k} x\right)^{2}} .
$$

and $\tau=\tau^{*}$ is the root of the equation $\sum_{i=1}^{n} J_{i}(x)=\bar{J}$. In particular if $\alpha \leq 1 / 2$ this saddle point is unique and if $\alpha=1$ then the saddle point decision maker strategy is the uniform one $\mathcal{P}$.

\section{Independent fading channel gains: fairness of expectation}

In this section we assume that the fading channel gains are independent. Namely, with probability $p^{k}, k \in[1, K]$ the fading channel gain of the jammer on the channel of user $i$ is $h_{i}^{k}$ and the uncontrolled noise for user $i$ is $N_{i}^{0 k}$. With probability $q^{l}, l \in[1, L]$ the fading channel gain of user $i$ is $g_{i}^{l}$. As a payoff we consider the expected $\alpha$-fairness $(\alpha \geq 0)$ utility function of the SNIRs

$$
v(P, J)=\frac{1}{1-\alpha} \sum_{i=1}^{n}\left(\sum_{k=1}^{K} \sum_{l=1}^{L} p^{k} q^{l} \frac{g_{i}^{l} P_{i}}{N_{i}^{0 k}+h_{i}^{k} J_{i}}\right)^{1-\alpha} \quad \text { for } \alpha \neq 1
$$

and

$$
v(P, J)=\sum_{i=1}^{n} \ln \left(\sum_{k=1}^{K} \sum_{l=1}^{L} p^{k} q^{l} \frac{g_{i}^{l} P_{i}}{N_{i}^{0 k}+h_{i}^{k} J_{i}}\right) \text { for } \alpha=1 .
$$

It is clear these payoffs are equivalent to the following ones:

$$
v(P, J)=\frac{1}{1-\alpha} \sum_{i=1}^{n}\left(\sum_{k=1}^{K} p^{k} \frac{P_{i} \bar{g}_{i}}{N_{i}^{0 k}+h_{i}^{k} J_{i}}\right)^{1-\alpha} \text { for } \alpha \neq 1
$$


and

$$
v(P, J)=\sum_{i=1}^{n} \ln \left(\sum_{k=1}^{K} p^{k} \frac{\bar{g}^{l} P_{i}}{N_{i}^{0 k}+h_{i}^{k} J_{i}}\right) \text { for } \alpha=1
$$

where

$$
\bar{g}_{i}=\sum_{l=1}^{L} q^{l} g_{i}^{l}
$$

The next theorem supplies the saddle point strategies.

Theorem 5 Let $0<\alpha \leq 1$ then the saddle point decision maker strategy is given as follows

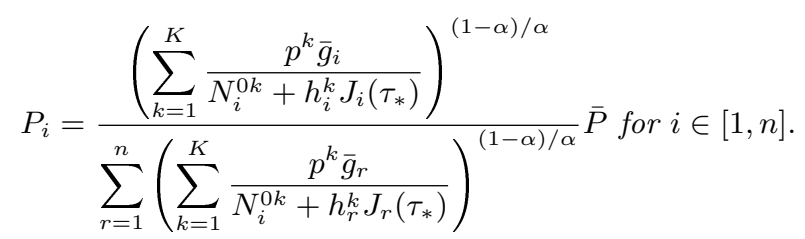

The saddle point jammer strategy is given by

$$
J_{i}(\tau)= \begin{cases}\text { the root of } R_{i}(x)=\tau, & \text { if } R_{i}(0)>\tau \\ 0, & \text { if } R_{i}(0) \leq \tau\end{cases}
$$

where

$$
R_{i}(x):=\left(\sum_{k=1}^{K} \frac{p^{k} \bar{g}_{i}}{N_{i}^{0 k}+h_{i}^{k} x}\right)^{1 / \alpha-2} \sum_{k=1}^{K} \frac{p^{k} h_{i}^{k} \bar{g}_{i}}{\left(N_{i}^{0 k}+h_{i}^{k} x\right)^{2}} .
$$

and $\tau=\tau^{*}$ is the root of the equation $\sum_{i=1}^{n} J_{i}(x)=\bar{J}$. In particular, if $\alpha \leq 1 / 2$ this saddle point is the unique one and if $\alpha=1$ then the saddle point decision maker strategy is the uniform one $\mathcal{P}$.

\section{The decision maker does not know if the jammer is present: expected fairness}

In this section we consider the power resource allocation problem where in the environment a jammer could either be present, bringing extra background noise to the natural one, or be absent. The decision maker has no exact knowledge about either presence or absence of the jammer. Namely, it knows that in the environment only a natural background noise could be with probability $\gamma$ meanwhile with probability $1-\gamma$ a jammer could come into the action distributing an extra noise of the total power $\bar{J}$ among users. The decision maker payoff is given as follows:

for $\alpha \neq 1$ :

$$
v_{P}(P, J)=\frac{1}{1-\alpha} \sum_{i=1}^{n}\left(\gamma\left(\frac{g_{i} P_{i}}{N_{i}^{0}}\right)^{1-\alpha}+(1-\gamma)\left(\frac{g_{i} P_{i}}{N_{i}^{0}+h_{i} J_{i}}\right)^{1-\alpha}\right),
$$


for $\alpha=1$ :

$$
v_{P}(P, J)=\sum_{i=1}^{n}\left(\gamma \ln \left(\frac{g_{i} P_{i}}{N_{i}^{0}}\right)+(1-\gamma) \ln \left(\frac{g_{i} P_{i}}{N_{i}^{0}+h_{i} J_{i}}\right)\right) .
$$

As a cost function to the jammer we consider $\alpha$-fairness $(\alpha>0)$ utility function of the SNIRs. Thus, his payoff is equal to his cost function taken with minus sign and it is given as follows:

$$
v_{J}(P, J)=-\frac{1}{1-\alpha} \sum_{i=1}^{n}\left(\frac{g_{i} P_{i}}{N_{i}^{0}+h_{i} J_{i}}\right)^{1-\alpha} \text { for } \alpha \neq 1
$$

and

$$
v_{J}(P, J)=-\sum_{i=1}^{n} \ln \left(\frac{g_{i} P_{i}}{N_{i}^{0}+h_{i} J_{i}}\right) \text { for } \alpha=1
$$

We assume that all the fading channel gains $g_{i}, h_{i}$ and the power level of the uncontrolled noise $N_{i}^{0}$ for $i \in[1, n]$, the probabilities $\gamma$ which the system is in as well as the total power resource $\bar{P}$ of the decision maker as well as the total noise $\bar{J}$ induced by the jammer are fixed and known to both players.

We shall look for a Nash equilibrium (NE), that is, we want to find $\left(P^{*}, J^{*}\right) \in$ $A \times B$ such that

$$
v_{P}\left(P, J^{*}\right) \leq v_{P}\left(P^{*}, J^{*}\right), \quad v_{J}\left(P^{*}, J\right) \leq v_{J}\left(P^{*}, J^{*}\right) \text { for any }(P, J) \in A \times B,
$$

where $A$ and $B$ are the sets of all the strategies of the decision maker and the jammer, respectively. In particular, we shall show that the NE exists and is unique and we shall provide closed form analytic expressions for its calculation.

It is interesting that this non-zero-sum game is a particular case of the zerosum game from Section 4. Namely, for this situation we have two states, so $K=2$ with the same user fading channel gains $g_{i}^{1}=g_{i}^{2}=g_{i}$. The probability that the environment is in the first state is $p^{1}=\gamma$ and the probability that the environment is in the second state is $p^{2}=1-\gamma$. The jammer fading channel gains for the first state equals to zero, namely, $h_{i}^{1}=0$, while the jammer fading channel gains for the second state is positive $h_{i}^{2}=h_{i}$. Then the jammer payoff (25) turns into

$$
v_{J}(P, J)=-\frac{1}{1-\alpha} \sum_{i=1}^{n}\left(\gamma\left(\frac{g_{i} P_{i}}{N_{i}^{0}}\right)^{1-\alpha}+(1-\gamma)\left(\frac{g_{i} P_{i}}{N_{i}^{0}+h_{i} J_{i}}\right)^{1-\alpha}\right) .
$$

It is clear that the first part of (27) does not depend on $J$, thus it is equivalent to $(25)$.

The following result providing the equilibrium in the closed form holds.

Theorem 6 The game has the unique equilibrium $(P, J)$. 
(a) If $0<\alpha<1$ then the equilibrium decision maker strategy is given as follows:

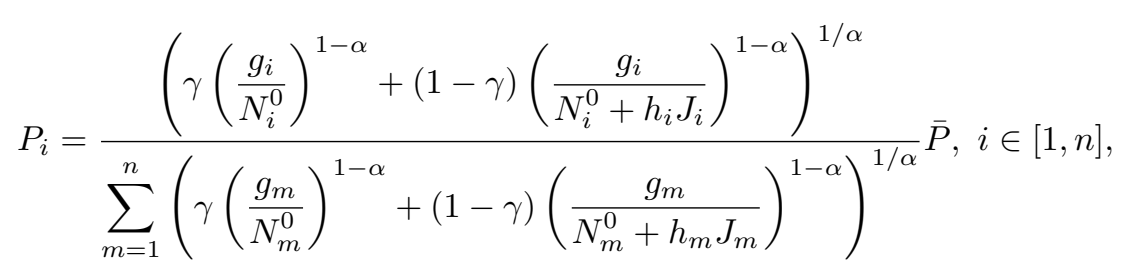

The equilibrium jammer strategy is $J_{i}=J_{i}(\tau)$ where

$$
J_{i}(\tau)= \begin{cases}\text { the unique root } x \text { of the equation } R_{i}(x)=\tau, & D_{i}>\tau, \\ 0, & D_{i} \leq \tau .\end{cases}
$$

where

$$
\begin{gathered}
R_{i}(x)=\left(\gamma\left(\frac{g_{i}}{N_{i}^{0}}\right)^{1-\alpha}+(1-\gamma)\left(\frac{g_{i}}{N_{i}^{0}+h_{i} x}\right)^{1-\alpha}\right)^{(1-\alpha) / \alpha} \frac{h_{i} g_{i}^{1-\alpha}}{\left(N_{i}^{0}+h_{i} x\right)^{2-\alpha}} \\
D_{i}:=R_{i}(0)=\frac{g_{i}^{(1-\alpha) / \alpha}}{\left(N_{i}^{0}\right)^{1 / \alpha}} h_{i}
\end{gathered}
$$

and $\tau=\tau^{*}$ is the unique root of the equation $\sum_{i=1}^{n} J_{i}(\tau)=\bar{J}$.

(b) If $\alpha=1$ then the equilibrium decision maker strategy is the uniform one $\mathcal{P}$. The equilibrium jammer strategy is $J_{i}=\left[1 / \nu-N_{i}^{0} / h_{i}\right]_{+}$where $\nu=\nu^{*}$ is the unique root of the following water-filling equation: $\sum_{i=1}^{n}\left[1 / \nu-N_{i}^{0} / h_{i}\right]_{+}=\bar{J}$.

\section{The decision maker does not know if the jammer is present: fairness of expectation}

In this section we consider a modification of the problem where the decision maker payoff is the fairness of expectation. So, the decision maker payoff is given as follows:

$$
v_{P}(P, J)=\frac{1}{1-\alpha} \sum_{i=1}^{n}\left(\gamma \frac{g_{i} P_{i}}{N_{i}^{0}}+(1-\gamma) \frac{g_{i} P_{i}}{N_{i}^{0}+h_{i} J_{i}}\right)^{1-\alpha} \text { for } \alpha \neq 1
$$

and

$$
v_{P}(P, J)=\sum_{i=1}^{n} \ln \left(\gamma \frac{g_{i} P_{i}}{N_{i}^{0}}+(1-\gamma) \frac{g_{i} P_{i}}{N_{i}^{0}+h_{i} J_{i}}\right) \text { for } \alpha=1 .
$$

The jammer still wants to minimize fairness of the resource allocation. The following result shows that the game has the unique equilibrium and it also supplies the equilibrium in the closed form. 
Theorem 7 The game has the unique equilibrium $(P, J)$.

(a) If $0<\alpha<1$ then the equilibrium decision maker strategy is given as follows:

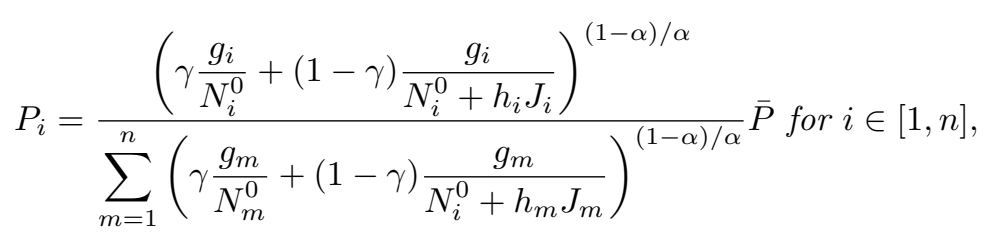

The equilibrium jammer strategy is $J_{i}=J_{i}(\tau)$ where

$$
J_{i}(\tau)= \begin{cases}\text { the unique root } x \text { of the equation } R_{i}(x)=\tau, & D_{i}>\tau \\ 0, & D_{i} \leq \tau\end{cases}
$$

where

$$
R_{i}(x)=\left(\gamma \frac{g_{i}}{N_{i}^{0}}+(1-\gamma) \frac{g_{i}}{N_{i}^{0}+h_{i} x}\right)^{(1-\alpha)^{2} / \alpha} \frac{h_{i} g_{i}^{1-\alpha}}{\left(N_{i}^{0}+h_{i} x\right)^{2-\alpha}}
$$

and $D_{i}$ is given by (30) (so, it is the same as for the expected fairness model) and $\tau=\tau^{*}$ is the unique root of the equation $\sum_{i=1}^{n} J_{i}(\tau)=\bar{J}$.

(b) If $\alpha=1$ then the equilibrium decision maker strategy is the uniform one $\mathcal{P}$. The equilibrium jammer strategy is $J_{i}=J_{i}(\nu)$ where

$$
J_{i}(\nu)= \begin{cases}\text { the unique root } x \text { of the equation } R_{i}^{1}(x)=\tau, & D_{i}^{1}>\nu \\ 0, & D_{i}^{1} \leq \nu\end{cases}
$$

where

$$
R_{i}^{1}(x)=\frac{(1-\gamma) h_{i} N_{i}^{0}}{\left(N_{i}^{0}+h_{i} x\right)\left(N_{i}^{0}+\gamma h_{i} x\right)}, \quad D_{i}^{1}:=R_{i}^{1}(0)=\frac{(1-\gamma) h_{i}}{N_{i}^{0}}
$$

and $\nu=\nu^{*}$ is the unique root of the equation: $\sum_{i=1}^{n} J_{i}(\nu)=\bar{J}$.

\section{Numerical examples for the game plots}

Here we apply the developed closed form solution to compare the saddle point strategies of the decision maker for the expected fairness game with dependent and independent plots and also their Jain's fairness indexes of $P$. Let there be five users, so $n=5$ and the system can be in two states $(K=2)$. Let the total power be $\bar{P}=3$, the background noise is permanent and $N_{i}^{0 k}=1$ for all $k$ and $i$. The optimal payoffs $v_{D}, v_{I}$ and the Jain's fairness indexes $\mathcal{J}_{\mathcal{D}}, \mathcal{J}_{\mathcal{I}}$ for dependent and independents plots are given in Table 1 as functions on $\alpha=0.1(0.2) 0.9$ and $p^{1}=0.0(0.1) 1.0$. We assume that $g=((5,1),(4,2),(3,3),(2,4),(1,5))$ and 
$h=((1,1),(1,1),(1,1),(1,1),(1,1))$ and for the independent plot we assume that $K=L q^{i}=p^{i}, i \in[1, K]$. We consider three cases of jamming power: (a) a small total jamming power $\bar{J}=0.1$ (Table 1 ), (b) a comparable total jamming power $\bar{J}=1$ with the base station power (Table 2) and (c) an overwhelming jamming power over the base station one $\bar{J}=30$ (Table 3 ).

It is interesting that the value of the game for dependent plot is less than or equal to the value of the game for independent plot while the Jain's fairness indexes have the opposite relations. The difference between the values of the game for two plots is decreasing in $\alpha$. The small total jamming power (Table 1) produces unnoticeable effect for big $\alpha$ compared to the optimization plot but decreasing $\alpha$ has impact on the value of the game as well as on the Jain's index of fairness. For overwhelming jamming power over the base station one the players tend to equalizing behavior but to switch to the equalizing behavior the independent plot requests larger jamming power threshold compared to the independent plots (Table 3). Equalizing behavior becomes earlier for large $\alpha$ then for small one (Table 2 and 3). Also, an increase in the total jamming power causes an increase of the Jain's fairness index.

Table 1. The case $\bar{J}=0.1$

\begin{tabular}{|c|c|c|c|c|c|c|c|c|c|c|c|c|}
\hline & & $p^{1}$ & $p^{1}$ & $p^{1}$ & $p^{1}$ & $p^{1}$ & $p^{1}$ & $p^{1}$ & $p^{1}$ & $p^{1}$ & $p^{1}$ & $p^{1}$ \\
\hline $\mathcal{J}_{v}$ & $\alpha$ & 0.0 & 0.1 & 0.2 & 0.3 & 0.4 & 0.5 & 0.6 & 0.7 & 0.8 & 0.9 & 1.0 \\
\hline $\mathcal{J}_{\mathcal{D}}$ & 0.1 & 0.33 & 0.37 & 0.44 & 0.56 & 0.79 & 1.00 & 0.79 & 0.56 & 0.44 & 0.37 & 0.33 \\
$\mathcal{J}_{\mathcal{D}}$ & 0.3 & 0.61 & 0.69 & 0.78 & 0.88 & 0.96 & 1.00 & 0.96 & 0.88 & 0.78 & 0.69 & 0.61 \\
$\mathcal{J}_{\mathcal{D}}$ & 0.5 & 0.84 & 0.88 & 0.93 & 0.97 & 0.99 & 1.00 & 0.99 & 0.97 & 0.93 & 0.88 & 0.84 \\
$\mathcal{J}_{\mathcal{D}}$ & 0.7 & 0.96 & 0.97 & 0.98 & 0.99 & 1.00 & 1.00 & 1.00 & 0.99 & 0.98 & 0.97 & 0.96 \\
$\mathcal{J}_{\mathcal{D}}$ & 0.9 & 1.00 & 1.00 & 1.00 & 1.00 & 1.00 & 1.00 & 1.00 & 1.00 & 1.00 & 1.00 & 1.00 \\
\hline$v_{D}$ & 0.1 & 12.02 & 11.21 & 10.46 & 9.80 & 9.32 & 9.16 & 9.32 & 9.80 & 10.46 & 11.21 & 12.02 \\
$v_{D}$ & 0.3 & 11.42 & 11.05 & 11.03 & 10.52 & 10.39 & 10.35 & 10.39 & 10.52 & 10.74 & 11.05 & 11.42 \\
$v_{D}$ & 0.5 & 13.21 & 13.08 & 12.98 & 12.91 & 12.87 & 12.86 & 12.87 & 12.91 & 12.98 & 13.08 & 13.21 \\
$v_{D}$ & 0.7 & 19.30 & 19.26 & 19.24 & 19.22 & 19.21 & 19.21 & 19.21 & 19.22 & 19.24 & 19.26 & 19.30 \\
$v_{D}$ & 0.9 & 52.27 & 52.27 & 52.27 & 52.26 & 52.26 & 52.26 & 52.26 & 52.26 & 52.27 & 52.27 & 52.27 \\
\hline \hline $\mathcal{J}_{\mathcal{I}}$ & 0.1 & 0.27 & 0.30 & 0.35 & 0.45 & 0.70 & 0.99 & 0.70 & 0.45 & 0.35 & 0.30 & 0.27 \\
$\mathcal{J}_{\mathcal{I}}$ & 0.3 & 0.60 & 0.68 & 0.77 & 0.87 & 0.96 & 1.00 & 0.96 & 0.87 & 0.77 & 0.68 & 0.60 \\
$\mathcal{J}_{\mathcal{I}}$ & 0.5 & 0.83 & 0.88 & 0.93 & 0.97 & 0.99 & 1.00 & 0.99 & 0.97 & 0.93 & 0.88 & 0.83 \\
$\mathcal{J}_{\mathcal{I}}$ & 0.7 & 0.96 & 0.97 & 0.98 & 0.99 & 1.00 & 1.00 & 1.00 & 0.99 & 0.98 & 0.97 & 0.96 \\
$\mathcal{J}_{\mathcal{I}}$ & 0.9 & 1.00 & 1.00 & 1.00 & 1.00 & 1.00 & 1.00 & 1.00 & 1.00 & 1.00 & 1.00 & 1.00 \\
\hline$v_{I}$ & 0.1 & 12.30 & 11.47 & 10.66 & 9.94 & 9.38 & 9.16 & 9.38 & 9.94 & 10.66 & 11.47 & 12.30 \\
$v_{I}$ & 0.3 & 11.44 & 11.06 & 10.75 & 10.53 & 10.39 & 10.36 & 10.39 & 10.53 & 10.75 & 11.06 & 11.44 \\
$v_{I}$ & 0.5 & 13.21 & 13.08 & 12.98 & 12.91 & 12.87 & 12.86 & 12.87 & 12.91 & 12.98 & 13.08 & 13.21 \\
$v_{I}$ & 0.7 & 19.30 & 19.26 & 19.24 & 19.22 & 19.21 & 19.21 & 19.21 & 19.22 & 19.24 & 19.26 & 19.30 \\
$v_{I}$ & 0.9 & 52.27 & 52.27 & 52.28 & 52.26 & 52.26 & 52.26 & 52.26 & 52.26 & 52.27 & 52.27 & 52.27 \\
\hline
\end{tabular}


Table 2. The case $\bar{J}=1$

\begin{tabular}{|c|c|c|c|c|c|c|c|c|c|c|c|c|}
\hline & & $p^{1}$ & $p^{1}$ & $p^{1}$ & $p^{1}$ & $p^{1}$ & $p^{1}$ & $p^{1}$ & $p^{1}$ & $p^{1}$ & $p^{1}$ & $p^{1}$ \\
\hline $\mathcal{J}_{v}$ & $\alpha$ & 0.0 & 0.1 & 0.2 & 0.3 & 0.4 & 0.5 & 0.6 & 0.7 & 0.8 & 0.9 & 1.0 \\
\hline $\mathcal{J}_{\mathcal{D}}$ & 0.1 & 0.59 & 0.62 & 0.72 & 0.86 & 0.99 & 1.00 & 0.99 & 0.86 & 0.72 & 0.62 & 0.59 \\
$\mathcal{J}_{\mathcal{D}}$ & 0.3 & 0.75 & 0.82 & 0.90 & 0.96 & 0.99 & 1.00 & 0.99 & 0.96 & 0.90 & 0.82 & 0.75 \\
$\mathcal{J}_{\mathcal{D}}$ & 0.5 & 0.89 & 0.93 & 0.96 & 0.99 & 1.00 & 1.00 & 1.00 & 0.99 & 0.96 & 0.93 & 0.89 \\
$\mathcal{J}_{\mathcal{D}}$ & 0.7 & 0.97 & 0.98 & 0.99 & 1.00 & 1.00 & 1.00 & 1.08 & 1.00 & 0.99 & 0.98 & 0.97 \\
$\mathcal{J}_{\mathcal{D}}$ & 0.9 & 1.00 & 1.00 & 1.00 & 1.00 & 1.00 & 1.00 & 1.00 & 1.00 & 1.00 & 1.00 & 1.00 \\
\hline$v_{D}$ & 0.1 & 8.95 & 8.55 & 8.20 & 7.98 & 7.91 & 7.91 & 7.91 & 7.98 & 8.20 & 8.55 & 8.95 \\
$v_{D}$ & 0.3 & 9.62 & 9.43 & 9.31 & 9.25 & 9.24 & 9.24 & 9.24 & 9.25 & 9.31 & 9.43 & 9.62 \\
$v_{D}$ & 0.5 & 11.95 & 11.90 & 11.87 & 11.85 & 11.85 & 11.85 & 11.85 & 11.85 & 11.87 & 11.90 & 11.95 \\
$v_{D}$ & 0.7 & 18.31 & 18.30 & 18.30 & 18.30 & 18.30 & 18.30 & 18.30 & 18.30 & 18.30 & 18.30 & 18.31 \\
$v_{D}$ & 0.9 & 51.42 & 51.42 & 51.42 & 51.42 & 51.42 & 51.42 & 51.42 & 51.42 & 51.42 & 51.42 & 51.42 \\
\hline \hline $\mathcal{J}_{\mathcal{I}}$ & 0.1 & 0.27 & 0.30 & 0.35 & 0.45 & 0.70 & 0.99 & 0.70 & 0.45 & 0.35 & 0.30 & 0.27 \\
$\mathcal{J}_{\mathcal{I}}$ & 0.3 & 0.64 & 0.72 & 0.80 & 0.89 & 0.97 & 1.00 & 0.97 & 0.89 & 0.80 & 0.72 & 0.64 \\
$\mathcal{J}_{\mathcal{I}}$ & 0.5 & 0.87 & 0.92 & 0.95 & 0.98 & 0.99 & 1.00 & 0.99 & 0.98 & 0.95 & 0.92 & 0.87 \\
$\mathcal{J}_{\mathcal{I}}$ & 0.7 & 0.97 & 0.98 & 0.99 & 0.99 & 1.00 & 1.00 & 1.00 & 0.99 & 0.99 & 0.98 & 0.97 \\
$\mathcal{J}_{\mathcal{I}}$ & 0.9 & 1.00 & 1.00 & 1.00 & 1.00 & 1.00 & 1.00 & 1.00 & 1.00 & 1.00 & 1.00 & 1.00 \\
\hline$v_{I}$ & 0.1 & 10.41 & 9.78 & 9.16 & 8.57 & 8.11 & 7.92 & 8.11 & 8.57 & 9.16 & 9.78 & 10.41 \\
$v_{I}$ & 0.3 & 9.83 & 9.61 & 9.46 & 9.34 & 9.27 & 9.24 & 9.27 & 9.34 & 9.46 & 9.61 & 9.83 \\
$v_{I}$ & 0.5 & 11.99 & 11.92 & 11.89 & 11.87 & 11.86 & 11.86 & 11.86 & 11.87 & 11.89 & 11.92 & 11.99 \\
$v_{I}$ & 0.7 & 18.31 & 18.30 & 18.30 & 18.30 & 18.30 & 18.30 & 18.30 & 18.30 & 18.30 & 18.30 & 18.31 \\
$v_{I}$ & 0.9 & 51.42 & 51.42 & 51.42 & 51.42 & 51.42 & 51.42 & 51.42 & 51.42 & 51.42 & 51.42 & 51.42 \\
\hline
\end{tabular}

\section{Conclusions}

In the present work we have dealt with the concept of alpha-fair resource allocation in the situation where the decision maker does not have complete information about the environment. Namely, we develop a concept of $\alpha$-fairness under uncertainty to allocate power resource in the presence of a jammer under two scenarios: (a) the decision maker does not have complete knowledge about the parameters of the environment but only knows their distribution, (b) the jammer can come into the environment with some probability bringing extra background noise. The goal of the decision maker is to maximize the $\alpha$-fairness utility function with respect to the SNIR. We have considered short-term fairness (the expected fairness) as well as long-term fairness (the fairness of expectation). We have modeled these plots using game-theoretical approaches since the jammer can be considered as an active agent acting against the decision maker. For all the plots the equilibrium strategies are found in closed form. We have shown that for all the scenarios the equilibrium has to be constructed in two steps. In the first step the equilibrium jamming strategy has to be constructed based on a solution of the corresponding modification of the water-filling equation. In the second step the decision maker equilibrium strategy has to be constructed equalizing the induced by jammer background noise. 
Table 3. The case $\bar{J}=30$

\begin{tabular}{|c|c|c|c|c|c|c|c|c|c|c|c|c|}
\hline & & $p^{1}$ & $p^{1}$ & $p^{1}$ & $p^{1}$ & $p^{1}$ & $p^{1}$ & $p^{1}$ & $p^{1}$ & $p^{1}$ & $p^{1}$ & $p^{1}$ \\
\hline $\mathcal{J}_{v}$ & $\alpha$ & 0.0 & 0.1 & 0.2 & 0.3 & 0.4 & 0.5 & 0.6 & 0.7 & 0.8 & 0.9 & 1.0 \\
\hline $\mathcal{J}_{\mathcal{D}}$ & 0.1 & 0.84 & 0.89 & 0.94 & 0.97 & 0.99 & 1.00 & 0.99 & 0.97 & 0.94 & 0.89 & 0.84 \\
$\mathcal{J}_{\mathcal{D}}$ & 0.3 & 0.89 & 0.93 & 0.96 & 0.98 & 0.99 & 1.00 & 0.99 & 0.98 & 0.96 & 0.93 & 0.89 \\
$\mathcal{J}_{\mathcal{D}}$ & 0.5 & 0.94 & 0.96 & 0.98 & 0.99 & 1.00 & 1.00 & 1.00 & 0.99 & 0.98 & 0.96 & 0.94 \\
$\mathcal{J}_{\mathcal{D}}$ & 0.7 & 0.97 & 0.98 & 0.99 & 1.00 & 1.00 & 1.00 & 1.00 & 1.00 & 0.99 & 0.98 & 0.97 \\
$\mathcal{J}_{\mathcal{D}}$ & 0.9 & 1.00 & 1.00 & 1.00 & 1.00 & 1.00 & 1.00 & 1.00 & 1.00 & 1.00 & 1.00 & 1.00 \\
\hline$v_{D}$ & 0.1 & 1.62 & 1.62 & 1.62 & 1.62 & 1.62 & 1.62 & 1.62 & 1.62 & 1.62 & 1.62 & 1.62 \\
$v_{D}$ & 0.3 & 2.69 & 2.69 & 2.69 & 2.69 & 2.69 & 2.69 & 2.69 & 2.69 & 2.69 & 2.69 & 2.69 \\
$v_{D}$ & 0.5 & 4.91 & 4.91 & 4.91 & 4.91 & 4.91 & 4.91 & 4.91 & 4.91 & 4.91 & 4.91 & 4.91 \\
$v_{D}$ & 0.7 & 10.78 & 10.78 & 10.78 & 10.78 & 10.78 & 10.78 & 10.78 & 10.78 & 10.78 & 10.78 & 10.78 \\
$v_{D}$ & 0.9 & 43.11 & 43.12 & 43.11 & 43.11 & 43.11 & 43.11 & 43.11 & 43.11 & 43.11 & 43.11 & 43.11 \\
\hline \hline $\mathcal{J}_{\mathcal{I}}$ & 0.1 & 0.27 & 0.30 & 0.35 & 0.45 & 0.70 & 0.99 & 0.70 & 0.45 & 0.35 & 0.30 & 0.27 \\
$\mathcal{J}_{\mathcal{I}}$ & 0.3 & 0.64 & 0.72 & 0.80 & 0.89 & 0.97 & 1.00 & 0.97 & 0.89 & 0.80 & 0.72 & 0.64 \\
$\mathcal{J}_{\mathcal{I}}$ & 0.5 & 0.88 & 0.92 & 0.95 & 0.98 & 0.99 & 1.00 & 0.99 & 0.98 & 0.95 & 0.92 & 0.88 \\
$\mathcal{J}_{\mathcal{I}}$ & 0.7 & 0.97 & 0.98 & 0.99 & 0.99 & 1.00 & 1.00 & 1.00 & 0.99 & 0.99 & 0.98 & 0.97 \\
$\mathcal{J}_{\mathcal{I}}$ & 0.9 & 1.00 & 1.00 & 1.00 & 1.00 & 1.00 & 1.00 & 1.00 & 1.00 & 1.00 & 1.00 & 1.00 \\
\hline$v_{I}$ & 0.1 & 2.10 & 1.96 & 1.83 & 1.82 & 1.62 & 1.58 & 1.62 & 1.71 & 1.83 & 1.96 & 2.10 \\
$v_{I}$ & 0.3 & 2.85 & 2.80 & 2.75 & 2.72 & 2.70 & 2.69 & 2.70 & 2.72 & 2.75 & 2.80 & 2.85 \\
$v_{I}$ & 0.5 & 4.95 & 4.93 & 4.92 & 4.92 & 4.91 & 4.91 & 4.91 & 4.92 & 4.92 & 4.93 & 4.95 \\
$v_{I}$ & 0.7 & 10.79 & 10.78 & 10.78 & 10.78 & 10.78 & 10.78 & 10.78 & 10.78 & 10.78 & 10.78 & 10.79 \\
$v_{I}$ & 0.9 & 43.11 & 43.11 & 43.11 & 43.11 & 43.11 & 43.11 & 43.11 & 43.11 & 43.11 & 43.11 & 43.11 \\
\hline
\end{tabular}

\section{References}

1. E. Altman, K. Avrachenkov, and A. Garnaev, "Jamming game in wireless networks with transmission cost", LNCS, v.4465, pp.1-12, Proc. of NET-COOP'07, 2007.

2. E. Altman, K. Avrachenkov and A. Garnaev, "Fair resource allocation in wireless networks in the presence of a jammer", in Proc. of ValueTools 2008, 2008.

3. G.T. Amariucai and S. Wei, "Jamming in Fixed-Rate Wireless Systems with Power Constraints - Part I: Fast Fading Channels". arXiv:0808.3431v1 [cs.IT], 2008.

4. G.T. Amariucai, S. Wei and R. Kannan, "Jamming in Fixed-Rate Wireless Systems with Power Constraints - Part II: Parallel Slow Fading Channels". arXiv:0808.3418v1 [cs.IT], 2008.

5. F.P. Kelly, "Charging and rate control for elastic traffic", European Trans. on Telecom., v.8, pp.33-37, 1998.

6. F.P. Kelly, A. Maulloo, and D. Tan, "Rate control for communication networks: shadow prices, proportional fairness and stability," Journal of the Operational Research Society, v.49, pp.237-252, 1998.

7. J. Mo and J. Walrand, "Fair end-to-end window-based congestion control", IEEE/ACM Trans. on Networking, v.8, pp.556-567, 2000.

8. S.H. Low, L. Peterson, and L. Wang, "Understanding TCP vegas: A duality model", in Proc. SIGMETRICS/Performance'01, pp.226-235, 2001.

9. Traffic management specification, The ATM forum Technical Committee, April 1996, version 4.0.

10. D. Tse and P. Viswanath, Fundamentals of Wireless Communication, Cambridge University Press, 2005. 